\title{
DEMO
}

Giellatekno Open-source click-in-text dictionaries for bringing closely related languages into contact.

\begin{abstract}
This is a demo of online dictionaries between minority languages with open-source FST morphology and lexical work. Open-source click-in-text dictionaries, NeahttaDigiSanit (NDS), have been developed in the Giellatekno infrastructure since 2012-2013. Originally, these dictionaries were implemented to provide a Samilanguage reading tool to provide click-in-text wordform recognition and translation for majority-language readers by which they could access text content written in minority languages. From the very beginning of 2013 this same dictionary has been localized not only for Sami languages, but for work with Balto-Finnic, Mordvin, Mari, Permic, Ugrian and Samoyedic languages, as well. There are now a number of NDS projects with Finnish, Hungarian, Russian and German majority langauge hubs. There are also a few instances of closely related language pairs outside of the Sami and Balto-Finnic branch; these include the Permic languages and Mordvin languages.
\end{abstract}

As of late October 2016, there are three sets of two-directional dictionaries operating between closely related languages: Udmurt<->Komi Zyrian; Komi Permyak<->Komi Zyrian, and Erzya <->Moksha.

\begin{tabular}{|l|l|l|l|}
\hline $\begin{array}{l}\text { Permic language } \\
\text { pairs }\end{array}$ & Source & Target & Lemmas \\
\hline http://kyv.oahpa.no & Udmurt & Komi Zyrian & 3427 \\
\cline { 2 - 4 } & Komi Zyrian & Udmurt & 5020 \\
\hline & Komi Permyak & Komi Zyrian & 1252 \\
\hline & Komi Zyrian & Komi Permyak & 1981 \\
\hline $\begin{array}{l}\text { Mordvin language } \\
\text { pairs }\end{array}$ & Erzya & Moksha & 3677 \\
\hline $\begin{array}{l}\text { http://valks.oahpa.n } \\
\text { o }\end{array}$ & Moksha & Erzya & 3613 \\
\hline
\end{tabular}

The lemmas and their glosses have been taken from the publications by Evgeni Tsypanov (Udmurt, Komi Permyak and Komi Zyrian), and Osip Polyakov (Moksha and Erzya). Open-source FST work comes directly projects in the Giellatekno infrastructure.

\section{Resources: \\ Dictionaries}

O.Е. Поляков 2004: Мокшень и эрзянь кяльхнень фкакс- и аф фкаксшисна. Синь валлувкссна. Эрзянь ды мокшонь кельтнень вейкекс- ды аволь вейкексчист. Сынст валлувост. / О.Е. Поляков, Ј. Rueter; Н.П. Огарёвонь лемса Мордовскяй государственнай университетсь. - Саранск: “Краснай Октябрь» типографиясь. - 264 с. https://victorio.uit.no/langtech/trunk/words/dicts/mdfmyv/ 
https://victorio.uit.no/langtech/trunk/words/dicts/myvmdf/

Цыпанов, Е.А. 1999: ПЕРЫМ-КОМИ ГИЖӦД КЫВ. Сыктывкар - «Пролог» небӧг ледзанін.

https://victorio.uit.no/langtech/trunk/words/dicts/kpvkoi/

https://victorio.uit.no/langtech/trunk/words/dicts/koikpv/

Цыпанов Й. 2007: Удмурт кыввор да лыддянкуд. Россияса наукаяс

академия Урал юкӧд Коми туялан шӧрин Кыв, литература да

история институт. - Сыктывкар.

https://victorio.uit.no/langtech/trunk/words/dicts/udmkpv/

https://victorio.uit.no/langtech/trunk/words/dicts/kpvudm/

Open-source FSTs:

https://victorio.uit.no/langtech/trunk/langs/koi/

https://victorio.uit.no/langtech/trunk/langs/kpv/

https://victorio.uit.no/langtech/trunk/langs/mdf/

https://victorio.uit.no/langtech/trunk/langs/myv/

https://victorio.uit.no/langtech/trunk/langs/udm/ 